\title{
Intensive ambulante Expositionsbehandlung bei schweren Zwängen - zwei Modelle aus der Praxis für die Praxis
}

\author{
Thomas Hillebrand $^{\mathrm{a}} \quad$ Nico Niedermeier ${ }^{\mathrm{b}}$ \\ aPraxis für Verhaltenstherapie, Münster, Deutschland

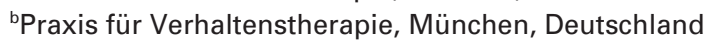

\section{Schlüsselwörter}

Zwangsstörung · Exposition mit Reaktionsverhinderung - Ambulante Verhaltenstherapie . S3-Leitlinien

\section{Zusammenfassung}

Die Behandlung der Zwangsstörung umfasst auch im ambulanten Therapiesetting die Reizkonfrontation mit Reaktionsverhinderung (Expositionsbehandlung) als zentrales Therapieelement. In der Versorgungsrealität wird sie jedoch kaum leitliniengerecht angewendet. Dieser Artikel analysiert daher zahlreiche Hinderungsgründe und nennt Vorschläge zu deren Überwindung. Vor dem Hintergrund langjähriger Praxiserfahrung der Autoren werden zudem konkrete Hinweise zur Durchführung einer intensiven Exposition in der ambulanten Behandlungssituation beschrieben.

Die intensive Expositionsbehandlung als zentrales Therapieelement in der Behandlung von Zwangsstörungen

Bis in die 1980er Jahre hinein galt die Zwangsstörung als eine schwer und häufig unzureichend behandelbare Störung. Erst durch die Einführung verhaltenstherapeutischer Expositionsverfahren sowie durch den Nachweis der Wirksamkeit des Antidepressivums Clomipramin bei Zwangsstörungen wandelte sich dieses Bild. Mittlerweile existieren zahlreiche Einzelstudien sowie Metaanalysen [Abramowitz, 1996; RosaAlcázar et al., 2008], die die Wirksamkeit einer kognitiv-behavioralen Therapie mit den verschiedenen Formen der Exposi-

\author{
Keywords \\ Obsessive-compulsive disorder . Exposure and \\ response prevention - Outpatient behavioral therapy . \\ Guidelines
}

\section{Summary}

Intensive Outpatient Exposure in the Treatment of Severe OCD - Two Models Gained in Practice, for Use in Practice Exposure and response prevention is the core element in OCD treatment, also in an outpatient setting. However, a guideline-based treatment is rarely applied in daily routine. This article outlines some of the numerous obstacles and provides several recommendations to overcome them. Based on their substantial experience in practice, the authors provide specific examples on how to conduct intensive exposure and response prevention in an outpatient setting.

tion bei Zwangsstörungen nachweisen konnten. In den verschiedenen Studien werden Symptomreduktionen von 51 bis $71 \%$ berichtet [Foa und Kozak, 1995; Foa et al., 2005]. Aufgrund dieser hohen empirischen Evidenz wurde die kognitive Verhaltenstherapie (KVT) mit Expositionsverfahren 2006 in die Leitlinien des National Institute for Health and Care Excellence (NICE) [National Collaborating Centre for Mental Health, 2006] und 2007 in die Leitlinien der American Psychological Association [APA, 2007] als wesentliches Therapieelement zur Behandlung von Zwangsstörungen aufgenommen.

Mit der Einführung der S3-Leitlinien für die Behandlung von Zwangsstörungen 2013 wird nun auch in Deutschland ein

\section{KARGER \\ Fax +497614520714 \\ Information@Karger.com}

www.karger.com (c) 2014 S. Karger GmbH, Freiburg

1016-6262/14/0243-0201\$39.50/0

Accessible online at:

www.karger.com/ver
Dipl.-Psych. Thomas Hillebrand

Praxis für Verhaltenstherapie

Hohenzollernring 67, 48145 Münster, Deutschland

praxis.hillebrand@gmx.de 
normatives Element für die Behandlung von Zwangsstörungen definiert, an dem sich Psychotherapeuten in ihrer Behandlung ausrichten sollen; ungeachtet der Frage, ob diese im ambulanten oder im stationären Rahmen stattfindet. Unter den Psychotherapieverfahren erreicht die KVT mit Exposition und Reaktionsmanagement in diesen Leitlinien den höchsten Empfehlungsgrad A. (Soll-Empfehlung: zumindest eine randomisierte kontrollierte Studie von insgesamt guter Qualität und Konsistenz, die sich direkt auf die jeweilige Empfehlung bezieht und nicht extrapoliert wurde). Ergänzt wird diese zentrale Aussage um Erläuterungen zu den konkreten Durchführungsmodalitäten, die den Empfehlungsgrad «klinischer Konsenspunkt» (KKP) erhalten, d.h. «empfohlen als gute klinische Praxis im Konsens und aufgrund der klinischen Erfahrung der Leitliniengruppe, bei dem keine experimentelle wissenschaftliche Erforschung möglich oder angestrebt ist» [Leitlinienreport zur S3-Leitlinie Zwangsstörungen, 2013]:

\section{Therapeutenbegleitung}

In der KVT sollen die Expositionen in Therapeutenbegleitung angeboten werden und auf eine Überführung in das Selbstmanagement des Patienten abzielen.

\section{Durchführung im störungsrelevanten Umfeld}

Expositionen im Rahmen einer KVT sollen von Therapeuten im häuslichen Umfeld oder in zwangsauslösenden Situationen (außerhalb der Praxis/Klinik) durchgeführt werden, falls die Zwangssymptome im Praxis- bzw. Kliniksetting nicht reproduzierbar sind.

\section{Dauer der Behandlung}

Die KVT mit Exposition und Reaktionsmanagement sollte in ihrer Intensität und Dauer den individuellen Gegebenheiten angepasst und bis zum Erreichen einer klinischen Besserung fortgeführt werden (Y-BOCS-Reduktion um mindestens $50 \%$, Verbesserung der Lebensqualität).

Angaben zur konkreten Dauer und Häufigkeit der jeweiligen Expositionsübung enthalten die S3-Leitlinien nicht. Sie verweisen jedoch auf eine Analyse der Intensität des Therapeutenkontakts in der britischen NICE-Leitlinie: Je intensiver, d.h. häufiger der Kontakt zwischen Patient und Therapeut, desto effektiver erweist sich die Therapie. Behandlungen mit mehr als 30 Sitzungen erzielten die höchsten Effektstärken. In dieser Gruppe von Studien kamen therapeutenbegleitete Expositionen mit hoher Frequenz zum Einsatz. So führten beispielsweise Foa et al. [1984] die Exposition täglich über 3 Wochen mit einer Dauer von $2 \mathrm{~h}$ durch; Rosqvist et al. [2001] setzten 90-minütige Expositionen 2 Mal pro Woche über einen Zeitraum von 12 Wochen ein. Bereits vor 20 Jahren empfahl eine Praktikerin im deutschsprachigen Raum [Reimer, 1994], die Übungen täglich über einen Zeitraum von 14 Tagen, zu Beginn über 2-3 h, in der zweiten Woche über $1 \mathrm{~h}$, gemeinsam mit dem Patienten durchzuführen. Die Behand- lung war übrigens bereits zu diesem Zeitpunkt abrechnungstechnisch möglich [Hand, 1993a,b]. Abramowitz et al. [2003] kamen zu dem Ergebnis, dass 15 Expositionssitzungen von jeweils $2 \mathrm{~h}$ täglich über einen Zeitraum von 3 Wochen oder von je 2 Einheiten pro Woche über einen Zeitraum von 8 Wochen $\mathrm{zu}$ dem gleichen langfristigen Resultat führen. Foa et al. [2012] stellten schließlich fest, dass die optimale Häufigkeit von Expositionssitzungen erst noch ermittelt werden müsse.

Fasst man die Ergebnisse der Studien sowie der Leitlinien zusammen, so ergeben sich aus unserer Sicht folgende Anforderungen an eine ambulante Expositionsbehandlung:

a) Expositionen sollen - zumindest anfänglich - therapeutenbegleitet sein;

b) Expositionen sollen mehrstündig erfolgen;

c) Expositionen sollen mehrmals pro Woche erfolgen;

d) Expositionen sollen in der störungsrelevanten Umgebung stattfinden.

Eine Expositionsbehandlung, die diese Kernpunkte umfasst, bezeichnen wir im Folgenden als intensive Expositionsbehandlung. Eine solche Intervention - z.B. bei einem Zwangspatienten mit Waschzwang aus Angst vor Kontamination mit Keimen - würde erfolgen, wie im Folgenden kurz beschrieben.

\section{Beispiel einer intensiven Expositionsbehandlung im ambulanten Setting}

Therapeut und Patient treffen sich in der Wohnung des Patienten. Nach einer kurzen Besprechung der geplanten Übungen, die bereits in den vorausgehenden Therapiestunden ausführlich vorbesprochen wurden, fasst der Patient verschiedene Gegenstände an, die in seinen Augen mit gefährlichen Keimen kontaminiert sind. Er berührt anschließend sich selbst und andere bislang penibel sauber gehaltene Bereiche seiner Wohnung und widersteht damit dem Drang, die Berührung zu vermeiden oder sich bereits nach dem ersten Kontakt mit einem unsauberen Gegenstand die Hände zu waschen. In Gedanken und im Dialog mit dem Therapeuten verstärkt er hierbei noch seine Befürchtung, «völlig verseucht» zu sein, um sich dem Reiz «maximal» zu exponieren. Der Patient verspürt nun einen deutlichen Anstieg der Anspannung; der Therapeut verbalisiert dies und lässt sich die aufkommenden Ängste und Gefühle beschreiben. Der Patient wird immer wieder darin unterstützt, dem Waschen zu widerstehen und den Abfall der Spannung abzuwarten. Ist die Anspannung auf ein tolerables Maß abgesunken, wird ein neuer Gegenstand ausgewählt und eine bislang besonders sauber gehaltene Zone berührt. Weiterhin wird auf ein Waschen der Hände verzichtet. Nach etwa 2,5 h des Übens wird der Expositionsblock beendet; der Patient verzeichnet einen Rückgang der Angst und der Anspannung auf ein erträgliches Maß. Zudem ist er deutlich erschöpft. Er ist motiviert, ausschließlich vereinbarte Händewaschungen am Abend vorzunehmen. Die Gewissheit, den Therapeuten am nächsten Tag wiederzusehen, hat zur 
Expositionsbereitschaft deutlich beigetragen. Am nächsten Tag wird dieses Vorgehen wiederholt. Hierbei ermutigen die Erfolge des ersten Behandlungsblocks den Patienten, sich auch am nächsten Tag mit weiteren schwierigen Situationen zu konfrontieren. Am dritten Tag werden bereits bekannte Übungen nochmals durchgeführt und um weitere ergänzt. Im Anschluss an diese therapeutenbegleitete Phase der Exposition geht der Patient nun in die Phase der eigenständig gesteuerten Expositionen über und bespricht die Resultate wieder in den Therapiesitzungen in der Praxis des Therapeuten. Ist mit diesem Vorgehen noch keine ausreichende Stabilisierung eingetreten oder bestehen noch kleinere «Zwangsinseln», können weitere gemeinsam durchgeführte «Booster-Expositionen» indiziert sein.

\section{Aktuelle Versorgungsrealität der intensiven Expositionsbehandlung im ambulanten Rahmen}

Die Mehrzahl der Untersuchungen, die sich mit der Durchführung von Exposition bei Zwangsstörungen beschäftigen, wurde im stationären Setting psychiatrischer Universitätskliniken oder an spezialisierten psychosomatischen Kliniken durchgeführt [z.B. Reinecker und Zaudig 1995; Althaus et al., 2000]. Ein weiterer Teil der Studien stammt aus psychiatrischen oder psychologischen Hochschulambulanzen [z.B. Aigner et al., 2004; O'Connor et al., 2006]. So entsteht der Eindruck, dass zumindest schwere Zwangsstörungen suffizient nur im Rahmen einer stationären Therapie oder einer ambulanten Spezialeinrichtung zu behandeln seien. Bereits 1976 [Boersema et al., 1976] und 1982 [Emmelkamp, 1982] konnte jedoch nachgewiesen werden, dass eine kostengünstigere ambulante Verhaltenstherapie ebenso effektiv sein kann [Bossert-Zaudig et al., 2002]. In jüngeren Untersuchungen [z.B. Niedermeier et al., 2007] und Falldarstellungen [Bossert-Zaudig und v. Buxhoeveden, 2013] konnte dies auch im deutschsprachigen Raum gezeigt werden.

Trotz dieser ermutigenden Ergebnisse belegen Studien aus der Versorgungsrealität in Deutschland, dass therapeutenbegleitete Behandlungen von Zwangsstörungen mit mehrstündigen Konfrontationsverfahren außerhalb der Praxis des Therapeuten nur sehr selten Anwendung finden. Dies spiegelt sich sowohl bei der Befragung von Patienten wider [Böhm et al., 2008] als auch in der Befragung von Therapeuten [Roth et al., 2004]. Eine Erhebung unter 174 Psychotherapeuten im Großraum Freiburg i.Br. ergab, dass $44 \%$ der Therapeuten Expositionsbehandlungen mit Zwangspatienten durchführen [Külz et al., 2010]. Dieses Ergebnis mag zunächst zwar erfreulich erscheinen, jedoch zeichnet die in dieser Studie enthaltene Detailanalyse der Durchführungsmodalitäten ein konträres Bild: Nur 16,9\% der teilnehmenden Therapeuten gaben an, «gelegentlich oder meistens» eine Exposition über eine Dauer von bis zu 2,5 h durchzuführen. Auf die Frage, ob eine Exposition als Blockbehandlung an 3 Tagen pro Woche erfolgt, gaben nur 5,8\% der Behandler an, dies «gelegentlich» durchzuführen; «meistens» oder «immer» erfolge dies gar nicht. Alle zentralen Kriterien einer intensiven Expositionsbehandlung wurden offensichtlich nur von einer sehr kleinen Minderheit eingehalten. Stattdessen erfolgten Expositionen - wie die Autoren der Studie treffend zusammenfassen - «häufiger in-sensu, häufiger in den Praxisräumlichkeiten und selten mit den tatschlichen Auslösern, häufiger während Einzelsitzungen und häufiger einmal pro Woche» [Külz et al., 2010]. Auf Nachfrage zur Begründung des Verzichts auf diese Methode, gaben die Befragten an, «keine Erfahrung mit Exposition» zu haben; zudem sei die Methode «zu zeitaufwendig», «nicht effektiv» und/oder «zu belastend für den Patienten». Schließlich seien «andere Verfahren ausreichend».

Detaillierte Hinweise zu den Hintergründen der Vermeidung einer intensiven Exposition ergaben sich in Workshops und Seminaren zur Therapie bei Zwangsstörungen. In diesem Kontext begegneten wir niedergelassenen Kollegen, die in überwiegender Mehrheit der Durchführung von Expositionen bei Zwangspatienten in ihrer Praxis skeptisch gegenüberstehen. Unmittelbar wurde argumentiert, dass eine intensive Reizkonfrontation die zeitlichen und strukturellen Abläufe einer normalen psychotherapeutischen Bestellpraxis sprenge. Die Unsicherheit bezüglich dieses Verfahrens wird durch zahlreiche weitere Einwände dokumentiert. Unser Eindruck ist, dass diese Zweifel an der Durchführbarkeit der Methode viele Therapeuten veranlassen, auf eine ambulante Behandlung zu verzichten, diese eher «halbherzig» durchzuführen oder grundsätzlich einen stationären Aufenthalt zu empfehlen. Wir möchten daher die prinzipielle Realisierbarkeit einer solchen intensivierten Expositionsbehandlung im ambulanten Setting, ausgehend von den Erfahrungen zweier Schwerpunktpraxen, illustrieren. Bevor wir die praktischen Aspekte der Durchführung beleuchten, wenden wir uns den eben erwähnten Einwänden und Vorbehalten der Therapeuten zu. Denn in der grundsätzlichen Bereitschaft des Therapeuten, diese Behandlungsmethode anzubieten, liegt der Schlüssel für eine erfolgreiche Anwendung.

\section{Vorbehalte ambulanter Therapeuten und deren Überwindung}

\section{«Der zeitliche und strukturelle Aufwand einer intensiven Exposition ist nicht mit dem Rahmen einer 〈normalen〉 Bestellpraxis vereinbar.»}

Einer der am häufigsten genannten Gründe beklagt den hohen Struktur- und Zeitaufwand, der für eine intensive Expositionsbehandlung anzusetzen ist. Plant man als Minimum 3 Zeitblöcke pro Woche zu jeweils 3 Therapiestunden ein, die zudem außerhalb der Praxis stattfinden, so wird der übliche Routineablauf einer psychologischen Bestellpraxis in der Tat deutlich unterbrochen. 
Eine Lösung für dieses Problem wird darin geboten, dass Expositionsverfahren in der Regel über mehrere Wochen vorbereitet werden und die konkrete Terminplanung mittelfristig, d.h. 4-6 Wochen vor der eigentlichen Durchführung erfolgt. Damit können die übrigen Patienten rechtzeitig auf eine Unterbrechung der Therapie in dieser Woche oder eine Terminverschiebung (im Falle regelmäßig vergebener Termine) hingewiesen werden. Ferner können z.B. auch Ferienzeiten, in denen naturgemäß zahlreiche Patienten abwesend sind, gezielt für die Durchführung von Expositionen genutzt werden. Dies soll aber nicht darüber hinwegtäuschen, dass die Umsetzung einer intensiven Exposition im ambulanten Setting eine zu Beginn ungewohnte - aber lohnende - Mehrbelastung für den Behandler darstellt.

\section{«Die Dauer der Exposition ist nicht vorhersehbar.»}

Generell gilt, dass Expositionsübungen im Rahmen eines vorher vereinbarten Zeitrahmens von 3-4 Therapiestunden in der Regel gut planbar sind. Hierzu ist es hilfreich, darauf zu achten, dass gegen Ende des Zeitraums keine schwierigen Konfrontationen mehr stattfinden. Diese können dann zu Beginn des nächsten Übungstages in Angriff genommen werden. Gleichzeitig ist es gängige Praxis, die Expositionen an Randzeiten des Arbeitstages zu legen, um im Bedarfsfall über einen zeitlichen Puffer in die Mittagszeit oder das Arbeitsende zu verfügen. Besonders therapeutischen Anfängern in der Durchführung von Expositionstherapie gibt dieser Puffer zusätzliche Sicherheit. Ein weiterer praktikabler Umgang mit unerwartet hohen Anspannungen zum Expositionsende ist die Vereinbarung von Telefonkontakten am selben Tag. Erscheint die persönliche Anwesenheit des Therapeuten nicht mehr zwingend notwendig, kann der Therapeut den weiteren Spannungsverlauf auch als ersten Schritt in das Selbstmanagement telefonisch kommentieren und begleiten.

\section{"Eine intensive Exposition im ambulanten Rahmen ist nicht} durch die Krankenkassen abrechenbar.»

Die frühere Richtlinie des einheitlichen Bewertungsmaßstabs (EBM), der zufolge der Rahmen einer Verhaltenstherapie von maximal $2 \mathrm{~h}$ pro Woche nicht überschritten werden durfte, galt lange als zentraler Hemmschuh zur Durchführung einer intensiven blockweisen Expositionsbehandlung. Diese Richtlinien wurden jedoch bereits 2006 geändert, um den Anforderungen von Expositionsbehandlungen speziell bei Angst- und Zwangsstörungen gerecht zu werden. Die Definition des «fakultativen Leistungsinhalts» der EBM-Ziffern 35220 und 35221 wurde ergänzt um die Bestimmung: «(...) auch in mehrstündigen Sitzungen bei entsprechendem zweifachen, dreifachen oder vierfachen Ansatz der Gebührenordnungsposition 35220 gemäß $§ 23 b$ Abs. 1 Nr. 3 der Psychotherapie-Richtlinien und $\S 11$ Abs. 14 Psychotherapie-Vereinbarungen (...)». Gemäß dieser Ergänzung können Behandlungsblöcke von 3-4 h abgerechnet werden. Es empfiehlt sich, im Antrag an den Gutachter darauf hinzuweisen, dass ein Reiz- konfrontationstraining mit Reaktionsmanagement en bloc von z.B. $4 \mathrm{~h}$ an bis zu 3 aufeinanderfolgenden Tagen durchgeführt werden soll; ein entsprechender Passus kann aber auch im Formular des Kurzzeittherapieantrags aufgeführt werden.

«Ich habe zu wenig Erfahrung mit Zwangspatienten generell und der Durchführung von Expositionsverfahren im Speziellen.»

Betrachtet man die Zahlen der Kassenärztlichen Vereinigung Westfalen-Lippe (KVWL), die den Behandlern zur Verfügung gestellt werden, um die Diagnosehäufigkeiten der eigenen Praxis mit dem Durchschnitt aller anderen Praxen im selben KV-Bezirk zu vergleichen, so zeigt sich, dass in dieser Region die Diagnose Zwangsstörung F42.1 mit 0,29\% lediglich Platz 22 der 25 häufigsten Diagnosen einnimmt (Quartal 3/2013). Die Ursachen für diese geringe Quote sind sicherlich vielfältig und liegen auch im Zögern zahlreicher Betroffener, eine Therapie aufzunehmen, begründet. Ein weiterer Grund könnte in der Haltung einiger Behandler liegen, Zwangspatienten und deren Problematik als «hartnäckig», «schwer behandelbar» und «nervenzehrend» zu erleben. Nicht selten wird die Vermutung geäußert, dass einige Therapeuten die Behandlung von Zwangsstörungen aus diesem Grunde vermeiden [Külz et al., 2010]. So haben wir es mit einem Teufelskreis zu tun: Wenig Erfahrung führt zur Vermeidung von Zwangspatienten, ein Erfahrungszuwachs bleibt aus.

Dieser Vermeidungsaspekt betrifft aber offenbar auch einen Großteil der Therapeuten, die prinzipiell bereit sind, sich der Behandlung dieser Störung zu stellen. Auch in dieser Gruppe führen, wie oben dargestellt, nur die wenigsten eine leitliniengerechte Exposition durch. Der Faktor «mangelnde Erfahrung mit Expositionsverfahren» - trotz grundsätzlichem Vertrauen in die Wirksamkeit dieser Methode - könnte hier eine zentrale Rolle spielen. Vielfach haben motivierte Therapeuten im Rahmen ihrer Ausbildung keine oder nur wenig praktische Erfahrung in der Durchführung von Exposition bei Zwangspatienten sammeln können. Sie sind daher unsicher, wie das Verfahren im Detail aufgebaut wird und befürchten, durch therapeutische Fehler die Symptomatik zu verschlimmern oder eventuell auftretenden intensiven Gefühlsreaktionen der Patienten nicht mehr Herr werden zu können. Zu Recht betonen Külz et al. [2010] den besonderen Schwierigkeitsgrad der Durchführung einer Exposition bei Zwängen im Vergleich zu einfachen Phobien. Als ein wichtiger Aspekt sei hier der bislang kaum beachtete Einfluss des für die Zwangsstörung spezifischen «Unvollständigkeitsgefühls» [Ecker und Gönner, 2006; Ecker et al., 2013; Summerfeldt, 2004] im Rahmen einer Exposition genannt. Diese Unsicherheiten können überwunden werden, wenn wir uns an diesem Verfahren «versuchen» und hierdurch Routine und Sicherheit aufbauen. Hilfreich erscheint es uns, hierzu entsprechende Seminarangebote wahrzunehmen. 
«Zwangsstörungen sind so hartnäckig, ich kann mir nicht vorstellen, dass eine Exposition wirklich helfen würde.»

Hinter diesem Vorbehalt steht ein genereller Zweifel an der Methode. Dass eine Expositionsbehandlung eine anhaltende Symptomveränderung bewirken kann, ist trotz evidenzbasierter Studienlage nicht vorstellbar. Die Gründe hierfür scheinen vielfältig. Zum einen kann eine grundsätzlich kritische Haltung gegenüber einer rein symptomorientierten Vorgehensweise vorliegen. Zum anderen erwächst dieser Vorbehalt nach unserer Erfahrung aus der «halbherzigen» Durchführung von Expositionen (z.B. Durchführung einer ausschließlich einmaligen Exposition, über eine Doppelstunde in der Praxis). Dies kann zu Misserfolgen führen, die den therapeutischen Pessimismus weiter verstärken. Hier steht der Therapeut motivational an einer ähnlichen Position wie der Zwangspatient, der zweifelt, ob er sich auf diese Methode, die zunächst einen Anstieg der Angst vorhersagt, einlassen soll.

Abhilfe schafft hier letztlich nur die Entscheidung zur Durchführung der Exposition, unterstützt durch das Vertrauen in die Studienlage und seit Kurzem in die Empfehlungen der Behandlungsleitlinien. Wie im vorgenannten Punkt, könnte hier der Besuch entsprechender Fortbildungsveranstaltungen helfen, weitere Zweifel auszuräumen und Sicherheiten aufzubauen.

Neben der Durchführung der Exposition ist als zweite Stellschraube die Erwartung an den Therapieerfolg zu überprüfen. Zwangserkrankungen lassen sich in den seltensten Fällen komplett heilen. Es gibt auch nach einer erfolgreichen Expositionsbehandlung häufig eine bestehende leichte Restsymptomatik. Es gilt also, eine realistische Erwartung des Therapieerfolgs zu etablieren, der in den Leitlinienempfehlungen als «Erreichen einer klinischen Besserung» beschrieben und als «Y-BOCS-Reduktion um mindestens 50\%, Verbesserung der Lebensqualität» definiert wird.

«Überschreitet man nicht die Grenzen einer therapeutischen Beziehung, wenn man so intensiv mit einem Patienten in so ungewohnter Umgebung arbeitet?»

Viele Therapeuten befürchten, dass durch die Expositionsbehandlung die therapeutische Beziehung beeinträchtigt werden könnte. Vielfach äußern sie die Befürchtung, den Patienten zu «verschrecken», indem sie zu tief in seine Privatsphäre eindringen. Insbesondere die Vorstellung, den Patienten in seinem häuslichen Umfeld aufzusuchen, scheint zahlreiche Psychotherapeuten zu verunsichern. In der Tat ist es zunächst ungewohnt, den sicheren Rahmen der Praxis zu verlassen. Die Erfahrung zeigt jedoch, dass die klare Therapeuten-PatientenBeziehung auch außerhalb der Praxis bestehen bleibt. Gleichzeitig wissen Patienten, die die Therapierationale der Exposition verstanden haben, in der Regel selbst genau, dass die wirksamste Konfrontation mit dem Zwang dort stattfindet, wo dieser sie am meisten «quält». Der Therapeut wird daher als professioneller Unterstützer verstanden, der schwierige Expositionserfahrungen überhaupt erst möglich macht. Patienten wissen diesen Einsatz des Therapeuten sehr zu schätzen und sind zumeist dankbar, einen Behandler zu finden, der sich bereit erklärt, diesen Weg gemeinsam mit ihnen zu gehen.

«Ist die Begleitung des Patienten bei den Übungen überhaupt sinnvoll? Sollte er das nicht alleine, in Eigenverantwortung durchführen?»

Hinter diesem Einwand verbirgt sich in der Regel ein mangelndes Verständnis für die Intensität, die die Zwangssymptomatik annehmen kann. Eine eigenständige Exposition ist dem Patienten unter solchen Umständen nicht möglich. Hier wird mitunter das Wesen des Zwangs verkannt und es existieren Veränderungszuschreibungen wie: «Wenn er wirklich will, dann kann er das auch allein.» Die therapeutenbegleitete Exposition hat dagegen den Sinn, dass der Patient schwierige Verhaltensweisen oft nach jahrelangem Vermeidungsverhalten erstmalig wieder realisiert und die zentrale Erfahrung der Habituation erlebt. Dies ist die Voraussetzung für weiterführende Übungen ohne Therapeuten.

\section{«Führt die Exposition nicht zu einer emotionalen}

Überlastung des Patienten, die ich dann nicht mehr

beherrschen kann?»

Manche Therapeuten befürchten, den Patienten im Rahmen der Exposition einer zu großen Belastung auszusetzen und eine emotionale Krise auszulösen, die sie im weiteren Verlauf nicht mehr kontrollieren können. Das Auslösen der hohen physiologischen Spannung ist natürlich Teil der Behandlungsrationale und daher gewünscht. Um diese Anspannung «handhabbar» werden zu lassen, kann auf der einen Seite ein graduiertes Vorgehen gewählt werden, bei dem die zu Beginn gewählten Auslösesituationen in einem mittleren Schwierigkeitsgrad erfolgen. Der Patient kann hier erste Habituationserfahrungen machen und Vertrauen in die Methode aufbauen. Ein weiterer Aspekt liegt in der kognitiven Vorbereitung, in der der Patient sich auf die Belastung, die auf ihn zukommt, einstellen kann. Ganz zentral für eine Anspannung, die ausschließlich expositionsinduziert ist, ist die freiwillige Entscheidung des Patienten zur Durchführung dieser Behandlung. Zu vermeiden ist ein zusätzlicher Spannungsanstieg in Form einer inneren Abwehr, die aus dem Gefühl entsteht, zur Exposition gedrängt worden zu sein. Diese kann in der Tat zu einer komplexen emotionalen Reaktion führen, die schwerer zu kontrollieren ist und in der Erfahrung eines Misserfolgs beim Patienten münden kann.

«Sollte sich der therapeutische Prozess nicht vielmehr mit den Hintergründen der Störung als mit dem Symptom befassen?»

Nicht nur bei tiefenpsychologisch ausgebildeten Psychotherapeuten findet sich bisweilen eine große Skepsis gegenüber sehr stark symptomorientierten Therapieelementen. Auch viele Verhaltenstherapeuten fokussieren in ihrer Arbeit die Hintergrundproblematik, also die vermuteten störungsauslösenden und aufrechterhaltenden Bedingungen, Famili- 
ensysteme, Kindheitserfahrungen, dysfunktionale Einstellungen, etc. Viele glauben insgeheim, dass sich die Zwangssymptomatik hierüber bessern wird. Diesem Glauben wird über neuere Therapieelemente der «dritten Welle» der Verhaltenstherapie wie z.B. Akzeptanz- und Commitmenttherapie, achtsamkeitsbasierte Therapieelemente oder schematherapeutische Ansätze zusätzlich Nährboden gegeben.

Auch wenn eine Klärung und Bearbeitung der Hintergrundbedingungen sowie der Funktionalität der Zwangsstörung unabdingbar ist und auch die genannten neuen Therapieelemente allesamt wichtige und sinnvolle Ergänzungen in unserer Arbeit sein können, stellt (zumindest nach derzeitiger Studienlage) bei Zwangsstörungen die intensive Exposition das wirksamste Therapieelement dar, ohne dessen Einsatz keine vergleichbaren Ergebnisse für die Betroffenen erreicht werden können.

Darüber hinaus bietet gerade eine intensive Expositionsbehandlung die Möglichkeit, sehr viel über die Persönlichkeit des Patienten, seine Lebensbedingungen, die Hintergründe der Störung, ihre Funktionalität und verschüttete Emotionen zu erfahren.

«Wenn ich im störungsrelevanten Bereich arbeite, werden meine Fahrtkosten nicht bezahlt.»

Dass sich im Detail eine finanzielle Benachteiligung einer externen Blockbehandlung im Vergleich zu Sitzungen in der Praxis ergibt, schildern ausführlich Bossert-Zaudig und v. Buxhoeveden [2013]. Sie kritisieren das Problem der nur ungenügend honorierten Fahrtzeit, in der ein in der Praxis verbleibender Therapeut eine honorierte Sitzung durchführen kann. Diese Problematik stellt in der Tat einen potenziell begrenzenden Faktor dar. Der Therapeut muss sich überlegen, wie viel Zeit er für einen Hausbesuch aufwenden möchte. Bis sich hier die Honorarsituation verbessert, sind wohl zweckdienliche Lösungen gefragt. Wenn der Anfahrtsweg zum Patienten morgens den Weg zur Praxis ersetzt und eine Rückkehr in die Praxis zur Mittagspause erfolgt, geht keine Sitzung verloren. Eine weitere pragmatische und sicherlich auch verhaltenstherapeutisch sinnvolle Vorgehensweise ist es, die Anund Abfahrten zu den jeweiligen Übungsorten gemeinsam mit dem Patienten zu unternehmen, hierbei kognitiv arbeitend die anstehende Übung vor- oder nachzubereiten, motivational tätig zu werden, oder in der Exposition aufkommende Gefühle nachzubesprechen. In diesem Fall wird der Weg zur Praxis zum unmittelbaren Teil der Therapie und hiermit im Rahmen des Stundenkontingentes abrechenbar.

\section{Gute Gründe, warum man im ambulanten Rahmen ein intensives Konfrontationstraining durchführen sollte}

In Anbetracht der seltenen und oft unzureichenden Durchführung einer intensiven Exposition im ambulanten Setting, könnte man die Fragen stellen, warum dieses Verfahren nicht spezialisierten stationären Einrichtungen vorbehalten bleibt. Unseres Erachtens gibt es zahlreiche Vorteile, die für eine Durchführung auch im ambulanten Rahmen sprechen.

\section{Vorteile für den Patienten}

\section{Hohe Erfolgswahrscheinlichkeit der Therapie}

Wie bereits mehrfach erwähnt, handelt es sich bei einer intensiven Exposition um ein Therapieelement, das aufgrund seiner empirisch gut gesicherten Wirksamkeit in die Leitlinien aufgenommen wurde. In Kombination mit KVT besteht für den Patienten darin eine hohe Wahrscheinlichkeit, dass sich seine Symptomatik bessert. Dieses Verfahren nicht anzuwenden, entspräche - scharf formuliert - einem Behandlungsfehler, beispielsweise zu vergleichen mit einem Internisten, der bei einem Magengeschwür auf die Gabe eines Protonenpumpenhemmers verzichten würde.

\section{Nähe zu relevanten Auslösern}

Ein wesentlicher Vorteil der ambulanten Exposition (gegenüber einer stationär durchgeführten Exposition) besteht in der Möglichkeit, dass sich Betroffene im Umfeld ihrer auslösenden und aufrechterhaltenden Bedingungen bewegen und diese hierüber leichter wahrgenommen und bearbeitet werden können. Sie sind also nicht wie in der Klinik ein aus der räumlichen Distanz retrospektiv gewonnenes Narrativ, sondern eine ständig beobachtbare, da sinnlich erfahrbare Einflussgröße der Therapie.

\section{Übbarkeit der Zwangssymptome}

Viele Zwangssymptome sind in ihrer Auslösung und in ihrer Übbarkeit an die Lebensumwelt des Patienten gebunden. So beziehen sich Wasch- und Kontrollzwänge häufig auf das häusliche Umfeld oder den Arbeitsplatz, aggressive Zwangsgedanken auf nahe Angehörige und Putzzwänge auf die eigene Wohnung. Diese Liste ließe sich weiter fortführen. Neben dem räumlichen Aspekt spielt oft auch der zeitliche Aspekt eine wichtige Rolle. Es gibt zahlreiche Zwänge, deren Auftreten oder Durchführung an bestimmte Tageszeiten gebunden ist. Als Beispiel sei ein Kontrollzwang in einer überfüllten U-Bahn zu Stoßzeiten genannt. Wie unschwer nachvollziehbar, ließe sich dieser Zwang nur schlecht im stationären Setting einer Klinik 100 km von der nächsten U-Bahn entfernt bearbeiten; gleichsam kann er natürlich auch nicht aus dem Therapiezimmer heraus im Rahmen der Sitzung in Angriff genommen werden.

\section{Flexibilität in der zeitlichen Planung}

Vorbereitung und Durchführung der Exposition sind zeitflexibel möglich, d.h. eine Exposition kann dann durchgeführt werden, wenn der Patient auch die notwendige Eigenmotivation und Freiwilligkeit aufbringen kann. Im Unterschied zum begrenzten stationären Aufenthalt, besteht kein Zeitdruck, 
der im ungünstigen Fall zur Exposition unter Druck und Zwang mit den entsprechenden limitierenden Effekten führen kann. In der ambulanten Praxissituation kann der Therapeut die notwendige Zeit zum Aufbau der Eigenmotivation aufbringen; im Einzelfall kann auch eine Therapieunterbrechung vereinbart werden, um sich an die Motivationslage anzupassen.

\section{Patienten mit prästationärer Symptomintensität}

Menschen mit leichten bis mittelschweren Zwängen, deren Alltagsbewältigung noch nicht elementar gestört ist und die einen stationären Aufenthalt noch nicht in Erwägung ziehen, profitieren von der ambulanten intensiven Exposition. Wenn nicht andere Gründe dafür sprechen, kann der stationäre Aufenthalt erspart bleiben.

\section{Vorteile für den Therapeuten}

Die Belohnungen, die für den Therapeuten mit dem Verfahren der intensiven Exposition einhergehen, finden viel zu selten Erwähnung. So ist die Exposition unserer Erfahrung nach einer der wenigen Momente, in dem Psychotherapeuten - vergleichbar mit einem chirurgischen Eingriff - in kurzer Zeit das Leben eines Menschen nachhaltig verändern können, indem rasch deutliche Symptomverbesserungen erzielt werden, die auf anderem Wege nicht zu erreichen sind. Daneben findet über die zeitliche, aber auch emotional dichte Arbeit zumeist eine starke Intensivierung der therapeutischen Beziehung statt und es entstehen weitere Einblicke in die Gesamtproblematik des Patienten. Auch können das Verlassen der Praxis und das Arbeiten unter den jeweils zwangsauslösenden Bedingungen eine erhebliche Abwechslung im Therapiealltag darstellen und somit nicht nur tief befriedigend, sondern auch stresspräventiv und auflockernd wirken.

\section{Hinweise zur Durchführung einer intensiven Expositionsbehandlung im ambulanten Setting}

Die grundsätzlichen Aspekte zur Vorbereitung und Durchführung einer Expositionstherapie bei Zwangserkrankungen sind in zahlreichen Lehrbüchern und Manualen ausführlich dargestellt [z.B. Förstner et al., 2011; Hoffmann und Hofmann, 2012; Lakatos und Reinecker, 2007]. Wir werden an dieser Stelle daher Aspekte fokussieren, die bei der Durchführung in der ambulanten Praxis von Bedeutung sind.

\section{Planung der Expositionstermine-}

\section{Blockierung im Terminkalender}

Ist der Patient soweit, sich eigenmotiviert auf das Expositionstraining einzulassen, lässt sich meist mit einem zeitlichen Abstand von 3-5 Wochen exakt bestimmen, wann die intensive Exposition stattfinden soll. Patienten können für diesen
Zeitraum Urlaub nehmen oder auch krankgeschrieben werden, damit sie sich auf den Prozess konzentrieren können. Die Zeit nach der Exposition stellt in der Regel eine vulnerable Phase für einen Rückfall dar. Hier gilt es, das Erreichte zu festigen. Daher sollten Expositionstrainings nicht vor den Urlaub des Therapeuten gelegt werden. Je näher die Exposition rückt, umso konkreter werden technisch-organisatorische Aspekte wie Anreise, Treffpunkt und Übungszeit besprochen.

\section{Zeit nehmen!}

Wie bereits erwähnt, ist es wichtig, gemeinsam genügend Zeit für die Übungsphase einzuplanen. Damit sich ein deutlicher Spannungsabfall einstellt, sollten zunächst mindestens 3 Therapiestunden (je nach therapeutischer Einschätzung mit der Möglichkeit, im Notfall noch Zeit anzuhängen) anberaumt werden, um jeglichen Zeitstress zu vermeiden. Zudem soll sich der Patient darauf verlassen können, dass zumindest an 2 Folgetagen der gleiche Zeitraum zur Verfügung steht. Die Gewissheit eines Folgetermins am nächsten Tag ist für den Patienten von entscheidender Bedeutung, sich auf Expositionen einzulassen, da er weiß, dass am nächsten Tag Erfahrungen besprochen werden können.

Ein weiterer Effekt der zeitnahen Wiederholung liegt im Aufbau einer realistischen Erwartung an den Erfolg des ersten Behandlungstages. Weder Patient noch Therapeut müssen den ersten Behandlungstag mit Erwartungen überfrachten, wie dies bei einer ausschließlich einmaligen Exposition der Fall wäre, und Enttäuschungen damit vorprogrammieren. Die Summe der anberaumten 3-4 Behandlungstage erzeugt den Effekt, dass erst zum Schluss Bilanz gezogen wird. Für Patienten ist die Veränderung, die sie vom ersten bis zum letzten Behandlungstag im Kontakt mit ihren kritischen Zwangsauslösern erleben, eine entscheidende und eindrückliche Erfahrung, die auf einem anderen Wege nicht erreicht werden kann.

\section{Schaffung einer günstigen Atmosphäre}

Abgesehen von den bereits erörterten Punkten wie beispielsweise der therapeutischen Beziehung, erscheint es uns wichtig, gerade im ambulanten Bereich darauf zu achten, dass z.B. bei einem Kontrollzwang einer jungen Mutter, der im eigenen Haus ausgeübt wird, nicht die kleinen Kinder zugegen sind oder der Ehemann ständig bei den Übungen zusieht. Gleiches gilt auch für Übungen in der Öffentlichkeit: Befürchtet ein Patient, «jemanden vor die U-Bahn stoßen zu können», ist es erforderlich, an einem vollen Bahnsteig zu üben. Um Befürchtungen zu verbalisieren und aufkommende - oft schambesetzte - Gefühle besprechen zu können, ist es hilfreich, sich in diesem Fall gemeinsam mit dem Therapeuten in eine abgelegene Ecke des Bahnhofs zurückziehen.

\section{Einbindung von Angehörigen}

Angehörige werden häufig von Betroffenen eingesetzt, um Zwangsbefürchtungen zu neutralisieren («Könntest Du bitte 
schnell nachsehen, ob der Herd auch wirklich aus ist, sonst werden wir kein schönes Wochenende auf Sylt verbringen können.»). Damit werden die Angehörigen instrumentalisiert und häufig zu Kobetroffenen. Gleichzeitig können sie auch eine zentrale Rolle in der Funktionalität der Zwangsstörung haben. Ein therapeutisches Einbeziehen der Angehörigen erscheint also zumeist sinnvoll. Dies bedeutet jedoch keinesfalls, dass sie als Kotherapeuten bei einer intensivierten Exposition eingesetzt werden sollten. Dies würden wir allenfalls bei Zwängen mit kleineren Kindern als Option erachten. Bei Erwachsenen sehen wir davon ab. Wenn die Angehörigen «mitgezwängelt» haben, sollten sie nicht abrupt nach einem Angehörigen-Gespräch jegliche Unterstützung verweigern, sondern abwarten, ob bzw. bis sich der Betroffene selbst, therapeutengeleitet, von ihrer Hilfe lossagt.

\section{Erneute Klärung der Verantwortlichkeit /}

\section{Unterbindung von Vermeidungsreaktionen}

$\mathrm{Zu}$ Beginn und während der Übungen weisen wir den Patienten wiederholt auf seine Eigenverantwortlichkeit und seine Ziele hin. In diesem Kontext gilt es auch, mögliches Vermeidungsverhalten als kontraproduktiv auszuweisen und während der Übung darauf zu achten, dass diese Verhaltensweisen nicht von dem Patienten eingesetzt werden. Sollten wir ihr Auftreten bemerken, machen wir den Patienten darauf aufmerksam und weisen erneut auf den Aspekt der Verantwortlichkeit hin. Ein Patient könnte beispielsweise kognitiv vermeiden, indem er denkt: «Na gut, dann verschmutze ich jetzt mein Bad, aber nach der Exposition werde ich es umso intensiver säubern». Wenn wir dieses Vermeidungspotenzial im Vorfeld thematisieren, wird es den meisten unserer Patienten gelingen, dieses Verhalten zu unterlassen bzw. bei dessen Auftreten unsere Hilfe einzufordern.

\section{Modellernen (guided mastering) zu Beginn der Behandlung} - Übergang in selbstständiges Üben

Insbesondere Menschen, die seit Jahren unter Zwängen leiden, haben oft den Eindruck, das Gefühl für einen «normalen» Umgang mit angst-/zwangsauslösenden Stimuli verloren zu haben. Hier ist es sehr hilfreich, wenn Therapeuten die Übungen zu Beginn modellhaft vorführen. In der nächsten Stufe soll der Betroffene die Übung im Beisein des Therapeuten umsetzen und mehrmals wiederholen. Erst, wenn er sicher ist, die Übung auch ohne Beisein des Therapeuten erfolgreich absolvieren zu können, wird der Patient ermutigt, diese (z.B. zwischen den tageweise gemeinsam angesetzten Übungseinheiten) selbständig zu wiederholen. Hier gilt es, dem Patienten Erfolge $\mathrm{zu}$ ermöglichen und nicht mehr zu fordern, als ihm zu diesem Zeitpunkt möglich ist.

\section{Exposition mit graduierter oder maximaler Reizexposition}

Da Erfolge für den Patienten motivierend sind, empfiehlt es sich, zunächst eine mittelschwere Übung gemeinsam durchzuführen, die der Patient sicher bewältigen kann. Ob dann stufenweise oder maximal exponiert wird, klären Patient und Behandler im Anschluss. Wir selbst exponieren sowohl graduiert als auch reizüberflutend und erleben aufgrund der langen Vorbereitungszeit und des Vertrauens der Patienten nur sehr selten Abbrüche der Exposition. Sinnvoll erscheint es uns auch, während eines Expositionsblocks möglichst mehrere Aspekte des Zwangs zu üben. Es sollte umfassend geübt werden, denn häufig fällt dem Patienten die Übung leichter, wenn sie im Vorfeld gemeinsam mit dem Therapeuten bearbeitet wurde.

\section{Kognitive Reattribuierung}

Bei der Durchführung eines Konfrontationstrainings gilt generell, dass spannungsauslösende Zwangsgedanken evoziert, neutralisierende Zwangshandlungen und Vermeidungsverhalten hingegen unterbunden werden. Befürchtungen sollen während des hohen Spannungsanstieges nicht kognitiv neutralisiert werden («Das ist ja gar nicht so schlimm»). Gleichzeitig wird im Rahmen der Exposition neben physiologischer Habituation auch eine kognitive Reattribuierung angestrebt. Nach dem Spannungsabfall, der durch die Exposition eintritt, wird metakognitiv - im Sinne der Zwangsdistanzierung - interveniert: «Es ist nur ein Gedanke, der nichts über die Realität aussagt».

\section{Nutzung der kathartischen Entblockung}

Häufig erleben Betroffene während der Exposition intensive Gefühle, die nicht unmittelbar der Übungssituation zuzuordnen sind. Beispielsweise kann ein Betroffener mit Waschzwang bei der Übung mit einem vermeintlich kontaminierten Gegenstand plötzlich tiefe Traurigkeit entwickeln. Hier kommen möglicherweise belastende Emotionen aus der Lebensgeschichte des Betroffenen zum Vorschein, die durch den Zwang gedeckelt wurden und nun einen Weg an die erlebte Oberfläche finden. Dieser Vorgang wird als «kathartische Entblockung» beschrieben. Diese Emotionen sollten keinesfalls übergangen werden, jedoch erscheint es auch nicht hilfreich, die Exposition nun völlig hin zur Bearbeitung der Hintergrundebene zu verlagern. Vielmehr sollen diese Emotionen wahrgenommen und verankert werden. Ihr Inhalt kann anschließend, unmittelbar nach der durchgeführten Übung, therapeutisch bearbeitet werden.

\section{Therapeutenverhalten:}

Konstatieren - Verbalisieren - Motivieren

Alle Übungen werden vom Therapeuten mit konstatierenden, verbalisierenden und motivierenden Interventionen begleitet. Immer wieder geht es darum, zu beschreiben, was gerade geübt wird und den Patienten zu ermuntern, sein emotionales und kognitives Erleben zu verbalisieren. Schließlich erfolgt eine regelmäßige Intensitätseinschätzung auf einer 10-stufigen Skala, unterschieden nach körperlicher Anspannung einerseits und emotionaler Befindlichkeit andererseits. Letztere kann bei Zwangspatienten aus Angst, Ekel oder 
Schuldgefühlen bestehen. Häufig werden auch eine als quälend empfundene innere Anspannung oder Unvollständigkeitsempfinden berichtet. Ziel dieser Interventionen ist eine Steigerung der Bewusstheit des Patienten, der die zahlreichen Eindrücke manchmal nur schwer verarbeiten kann. Es ist daher durchaus sinnvoll, mehrmals zu wiederholen, welche Übungen der Patient bereits erfolgreich bewältigt hat und dies mit einem Lob zu verbinden.

\section{Zwei Beispiele aus der Praxis}

Im Folgenden stellen wir exemplarisch 2 Modelle aus der ambulanten Versorgungsrealität dar, in denen die Behandlung von Zwangspatienten einen deutlichen Schwerpunkt darstellt.

\section{Modell aus Münster}

Die Praxis ist eine reguläre Einzelpraxis. Den Zeitraum eines Jahres (Mai 2013 - Mai 2014) als Querschnitt betrachtet, wurden 61 Patienten mit Zwangsstörungen in den unterschiedlichen Therapiestadien behandelt. Bei 29 Patienten wurde eine intensive Exposition durchgeführt, diese teilweise bereits vor diesem Jahreszeitraum. Jährlich finden im Durchschnitt ca. 10 intensive Expositionen statt. Bei 6 Patienten ist eine Exposition aktuell in Vorbereitung. 18 Patienten erreichten im Rahmen unbegleiteter Expositionen und einer zwangsspezifischen Therapie eine Symptomreduktion, bei 5 Patienten stand eine schizophrene Symptomatik im Vordergrund, 2 Patienten wurden in stationäre Behandlung überwiesen, eine Patientin ist aktuell poststationär sehr stabil und in der Therapieanfangsphase.

Der Expositionsblock wird zunächst auf 3 Tage mit jeweils $3 \mathrm{~h}$ pro Woche angesetzt. Danach werden Übungen zur Selbstexposition vereinbart und in den regulären Therapiesitzungen weiterbegleitet. Sind weitere therapeutenbegleitete Expositionsblöcke erforderlich, werden diese durchgeführt. Patienten, bei denen dieser Behandlungsumfang von vornherein nicht ausreichend erscheint, werden in den stationären Aufenthalt überwiesen. Dies ist z.B. dann der Fall, wenn die Zwangssymptomatik akut so stark ausgeprägt ist, dass keine Alltagsbewältigung mehr möglich ist. Die Exposition erfolgt in der Regel zu Beginn der Behandlung. Im Rahmen der Vorbereitung liegt der Schwerpunkt auf der Erarbeitung der verhaltenstherapeutischen Therapierationale bezüglich der Exposition, aber auch dem Verständnis der Zwangsstörung als eigenständige neurophysiologische Erkrankung, die nicht zwingend ein Symptom für z.B. einen Konflikt sein muss. Im Anschluss an die Exposition und eine deutliche Symptomreduktion wird in den meisten Fällen an parallel bestehenden Problemfeldern gearbeitet. Die Qualität der therapeutischen Beziehung nach der Exposition sowie zusätzliche Informationen, die mitunter im Rahmen der Exposition zutage treten, sind für diesen therapeutischen Prozess sehr frucht- bar. Es gibt aber auch Patienten, die nach erfolgreicher Exposition zufrieden mit der Symptomreduktion sind und - wie es einmal ein Patient formulierte - zunächst «Gewinnmitnahme» betreiben wollen. In diesen Fällen wird die Therapie nach erfolgreicher Exposition gemeinsam beendet [Hillebrand, 2006].

\section{Modell aus München}

Die Praxis umfasst eine Gemeinschaft aus 7 ärztlichen und psychologischen approbierten Psychotherapeuten, einem Psychiater und mehreren Ausbildungsassistenten. Derzeit werden ca. 70 Patienten mit Zwangsstörungen behandelt.

Jeder der Therapeuten führt pro Jahr durchschnittlich 4-8 intensive Reizkonfrontationstrainings im Rahmen einer multimodalen Zwangstherapie durch. Mehr als 8 intensive Expositionstrainings erscheinen uns im ambulanten Setting kaum durchführbar, da dies den regulären Praxisalltag zu sehr beeinträchtigen würde. Bei Jugendlichen (ab 16 Jahren) und Erwachsenen wird als Expositionsverfahren zumeist Reizüberflutung mit Reaktionsmanagement (Flooding) durchgeführt. Bei Kindern von 14-16 Jahren wird zumeist eine stufenweise Konfrontation durchgeführt. Die Mehrzahl der Patienten leidet unter schweren bis extremen Zwängen.

Die intensive Expositionstherapie wird nach 2- bis 6-monatiger Vorbereitung im Rahmen der Gesamttherapie, bei der auch vorrangig an den Hintergründen der Störung gearbeitet wird, als Block (2-5 aufeinanderfolgende Therapieeinheiten pro Tag) an 3-21 Tagen in der störungsrelevanten Umgebung des Patienten durchgeführt. Treten beispielsweise die Zwänge des Patienten nachts in der U-Bahn auf, wird nachts mit dem Patienten in der U-Bahn geübt; wenn sie morgens in der Wohnung aufkommen, werden sie entsprechend zur selben Zeit und am selben Ort bearbeitet.

Es wird versucht, so viele Tage hintereinander (wobei wir durchaus die Wochenenden pausieren) zu üben, bis die Symptomatik soweit gesunken und die Kompetenz und die Zuversicht des Patienten soweit angestiegen sind, dass ein Übergang in eine reine Konfrontation im Selbstmanagement Erfolg versprechend erscheint. Zwischen den therapeutengeleiteten Expositionen werden die Patienten jedoch bereits von Beginn an stets angehalten, im Selbstmanagement weiterzuüben.

Der zeitliche Umfang, der insgesamt mit einzelnen Patienten geübt wird, variiert sehr stark, je nach Bedarf des Patienten. Durchschnittlich wird über einen Zeitraum von 14,1 Therapiestunden (dies entspricht $11 \mathrm{~h}$ ) mit jedem Patienten geübt. Nach der Phase der intensiven Exposition verpflichten sich die Patienten, im Selbstmanagement weiterzuüben. Der Therapieschwerpunkt wechselt wieder hin zur Bearbeitung der Hintergründe der Störung.

Die Ergebnisse dieses Verfahrens [Niedermeier et al., 2007] legen nahe, dass mit einem intensivierten Vorgehen auch im ambulanten Bereich vergleichbar gute Ergebnisse erzielt werden können wie in einem auf Zwänge spezialisierten 
stationären Setting. Die Ergebnisse erwiesen sich nach durchschnittlich 31 Monaten Katamnese als stabil.

\section{Fazit}

Die Durchführung einer leitliniengerechten Behandlung von Zwangsstörungen mit einem therapeutenbegleiteten intensiven Konfrontationstraining ist auch in der ambulanten psychotherapeutischen Praxis möglich und unter Versorgungsaspekten notwendig. Voraussetzung ist die Bereitschaft des Therapeuten, bisherige Vorbehalte zu überdenken, gegebenenfalls Unsicherheiten durch gezielte Fortbildung und Supervision zu mindern und sich zur Durchführung dieser für den Patienten hochwirksamen Methode der intensivierten Exposition zu entscheiden. Gleichsam erscheint es notwendig, dass sich auch die Kostenträger verstärkt engagieren, indem sie dieses hocheffektive Verfahren durch Anreize in den Vergütungsmodellen (z.B. Honorierung von Anreisezeiten, Boni für geblockte auswärtig durchgeführte Expositionsbehandlungen oder Ähnliches) gezielt fördern. Insgesamt bedarf es zahlreicher weiterer ambulanter Psychotherapeuten, die sich zum Wohle der Patienten bereit erklären, ein solches Verfahren durchzuführen. Diese, so hoffen wir, konnten wir hierzu ermutigen.

\section{Disclosure Statement}

Die Autoren erklären hiermit, dass kein Interessenskonflikt in Bezug auf das Manuskript besteht.

\section{Literatur}

Abramowitz JS: Variants of exposure and response prevention in the treatment of obsessive-compulsive disorder: a meta-analysis. Behav Res Ther 1996;27:583-600.

Abramowitz JS, Foa EB, Franklin ME: Exposure and ritual prevention for obsessive-compulsive disorder: effects of intensive versus twice-weekly sessions. J Consult Clin Psychol 2003;71:394-398.

Aigner M, Dema I, Zitterl W, Bach M, Trappl E, Lenz G: Verhaltenstherapeutische Gruppentherapie für Zwangsstörungen. Verhaltenstherapie 2004;14: $7-14$

Althaus D, Zaudig M, Röper G, Butollo W: Wirksamkeit eines spezifisch für Zwangsstörungen entwickelten stationären Gruppentherapiekonzeptes bei gleichzeitiger Verhaltenstherapie und pharmakologischer Behandlung. Verhaltenstherapie 2000; 10;16-23.

American Psychological Association (APA): Guidelines 2007, Practice Guideline for the Treatment of Patients with Obsessive-Compulsive Disorder. www.psychiatryonline.org/content. aspx?bookid $=28 \&$ section $i d=1678180$ (Zugriff 12.08.14).

Böhm K, Förstner U, Külz A, Seer N, Voderholzer U: Versorgungsrealität der Zwangsstörungen: Werden Expositionsverfahren eingesetzt? Verhaltenstherapie 2008;18:18-24.

Boersema K, Hengst S, Bekker J, Emmelkamp PMG: Exposure and response prevention in the natura environment. A comparison with obsessive-compulsive patients. Behav Res Ther 1976;14:19-24.

Bossert-Zaudig S, v. Buxhoeveden I: Therapieempfehlung versus Realität. Anwendung und Kosten leitlinienbasierter Techniken von ambulanter Verhaltenstherapie bei Zwangsstörungen: Erfahrungen und Therapieprotokolle aus einer Routinepraxis. Ärztliche Psychotherapie 2013;8:30-38.

Bossert-Zaudig S, Zaudig M, Simon M: Empirische Befunde zur Effektivität der Verhaltenstherapie; in Zaudig M, Hauke W, Hererl U: Die Zwangsstörung. Stuttgart, Schattauer, 2002, pp 107-123.

Ecker W, Gönner, S: Das Unvollständigkeitsgefühl. Neuentdeckung eines alten psychopathologischen Symptoms bei Zwangserkrankungen. Der Nervenarzt 2006;77;1115-1122.
Ecker W, Kupfer J, Gönner S: Selbstbezogenes Unvollständigkeitserleben bei Zwangsstörungen. Verhaltenstherapie 2013;23:12-21.

Emmelkamp PMG: Phobic and Obsessive-Compulsive Disorders: Theory, Research and Practice. New York, Plenum Press, 1982.

Foa EB, Kozak MJ: DSM-IV field trial. Obsessivecompulsive disorder. Am J Psychiatry 1995;152: 90-96.

Foa EB, Liebowitz MR, Kozak MJ, et al.: Randomized placebo-controlled trial of exposure and ritual prevention, clomipramine, and their combination in the treatment of obsessive-compulsive disorder. Am J Psychiatry 2005;162;151-161.

Foa EB, Steketee G, Grayson JB, Turner RM, Latimer PR: Deliberate exposure and blocking of obsessive-compulsive rituals: immediate and long-term effects. Behav Ther 1984;15:450-472.

Foa EB, Yadin E, Lichner TK: Exposure and Response (Ritual) Prevention for Obsessive-Compulsive Disorder. New York, Oxford University Press, 2012.

Förstner U, Külz AK, Voderholzer U: Störungsspezifische Behandlung der Zwangsstörungen. Ein Therapiemanual. Stuttgart, Kohlhammer, 2011.

Hillebrand T: Konfrontationsbehandlung von Zwängen in der ambulanten Praxis; in Fricke S, Rufer M, Hand I (eds): Verhaltenstherapie bei Zwangsstörungen. München, Urban und Fischer, 2006, pp 120-132.

Hand I: Verhaltenstherapie für Zwangskranke und deren Angehörige; in Möller HJ (ed): Therapie psychiatrischer Erkrankungen. Stuttgart, Enke, 1993a.

Hand I: Mitteilungen der Verbände: Aktuelle Information zu Kassenleistungen bei Exposition in vivo. Verhaltenstherapie 1993b;3:141.

Hoffmann N, Hofmann B: Expositionen bei Ängsten und Zwängen, ed 3. Weinheim, Beltz, 2012.

Külz AK, Hassenpflug K, Riemann D, Linster HW, Dornberg M, Voderholzer U: Ambulante psychotherapeutische Versorgung bei Zwangserkrankungen. Ergebnisse einer anonymen Therapeutenbefragung. Psychother Psych Med 2010;60:194-201.
Lakatos A, Reinecker H: Kognitive Verhaltenstherapie bei Zwangsstörungen. Ein Therapiemanual, ed 3. Göttingen, Hogrefe, 2007.

Leitlinienreport zur S3-Leitlinie Zwangsstörungen, 2013. AWMF-Registernummer 038/017. www dgppn.de/fileadmin/user_upload/_medien/download/pdf/kurzversion-leitlinien/S3-Leitlinie Zwangsst $\%$ C3\% B6rungen_Leitlinienreport_Endversion_19_08_2013.pdf (Zugriff 07.08.2014).

National Collaborating Centre for Mental Health: Obsessive-compulsive disorder: core interventions in the treatment of obsessive-compulsive disorder and body dysmorphic disorder. Leicester, British Psychological Society, 2006.

Niedermeier N, Pfeiffer-Gerschel T, Manzinger H, Mangold W, Althaus D: Möglichkeiten und Grenzen der ambulanten Verhaltenstherapie bei Zwangsstörungen - Ergebnisse aus einer verhaltenstherapeutischen Praxis. Verhaltensther Verhaltensmed 2007;28:188-203.

O’Connor K, Aardema F, Robillard S, et al.: Cognitive behavior therapy and medication in the treatment of obsessive-compulsive disorder. Acta Psychiatr Scand 2006;113:408-419.

Reimer M, Tillmanns A, Tillmans I: Ambulante Verhaltenstherapie der Zwangsstörung in der kassenärztlichen Versorgung: Wichtige Therapieschwerpunkte aus der Erfahrung in der Praxis. Verhaltenstherapie 1994;4:266-274.

Reinecker H, Zaudig M: Langzeiteffekte bei der Behandlung von Zwangsstörungen. Lengerich, Pabst, 1995.

Rosa-Alcázar AI, Sánchez-Meca J, Gómez-Conesa A, Marín-Martínez F: Psychological treatment of obsessive-compulsive disorder: a meta-analysis. Clin Psychol Rev 2008;28:1310-1325.

Rosqvist J, Egan D, Manzo P, Baer L, Jenike MA, Willis BS: Home-based behavior therapy for obsessive-compulsive disorder: a case series with data. J Anxiety Disord 2001;15:395-400.

Roth C, Siegl J, Aufdermauer N, Reinecker H: Therapie von Angst- und Zwangspatienten in der verhaltenstherapeutischen Praxis. Verhaltenstherapie 2004;14:16-21.

Summerfeldt LJ: Understanding and treating incompleteness in obsessive-compulsive disorder. J Clin Psychol 2004;60:1155-1168. 